\title{
Classification of Melanoma Considering the Adaptive Additional Shape Feature
}

\author{
K. Chaiyakhan, P. Chujai, R. Chanklan, and N. Kerdprasop
}

\begin{abstract}
Skin cancer is the rapid growth of abnormal skin tissues. There are several types of skin cancer, and the deadliest being melanoma. The most important warning sign for melanoma is a lesion on the skin that keeps on developing in shape, color or size. Melanoma must be detected in the initial stage, for early medical treatment. In this research, we propose and approach to automatically classify melanoma. Otsu method was used to get the lesion region. A feature that is usually used in melanoma classification in visual checking was use. The additional feature that we also use is adaptive additional shape feature (ADPS) to specific changing of curve. In classification process we use deep neural networks for skin lesions classification. We also compare deep neural networks with support vector machine. The experimental results of classification demonstrated that ADPS with other features using deep neural network as classifier provided good classification in evaluation process.
\end{abstract}

Index Terms-Image segmentation, feature extraction, melanoma detection, skin cancer.

\section{INTRODUCTION}

A nevus is a benign or non-cancerous melanocytic tumor and can also be referred to as a mole. In general, moles will not cause any problems, but a person who has more than 5 atypical or irregular moles has more risk of developing melanoma [1], the major aggressive kind of skin cancer. More than 10,000 people are killed each year in the United States. Separating moles from melanoma is not easy, even for dermatologists with years of experience. Melanoma skin cancer can rapidly expand to other organs, detecting melanoma early is especially important in order to have chance for successful treatment.

Frequently, the initial stage of melanoma is a change in shape, size, or color of an existing mole. Regular moles and melanomas do not look the same. Generally, a normal mole has a perfectly round shape, while melanoma has an irregularly shape. The analysis of melanoma is completed by examination of skin lesions with an optical device called dermatoscope used by experienced dermatologists to obtain a magnification view of skin structure, this technique is called dermoscopy. Dermoscopy has a high sensitivity and

Manuscript received June 22, 2019; revised October 22, 2019.

K. Chaiyakhan is with the Computer Engineering Department, Rajamangala University of Technology Isan, Muang, Nakhon Ratchasima, Thailand (e-mail: kedkarnc@hotmail.com).

P. Chujai is with the Electrical Technology Education Department, Faculty of Industrial Education and Technology, King Mongkut's University of Technology Thonburi, Bangkok, Thailand (e-mail: pasapitchchujai@gmail.com).

R. Chanklan and N. Kerdprasop are with the School of Computer Engineering, Suranaree University of Technology, Nakhon Ratchasima, Thailand (e-mail: arc_angle@hotmail.com, nittaya.k@gmail.com). specificity for the diagnosis of melanoma [2]. Visual checking is used to detect melanoma based on ABCDE method, where A represents asymmetry, B represents border irregularity, C represents color variegation, D represents diameter and E represents evolution. Normally, for detecting melanoma from dermoscopy images the process can be separated into 5 steps: image acquisition, image pre-processing (enhancement, noise reduction and artifact filtering), lesion segmentation, feature extraction and classification. Computer Aided Melanoma Skin Cancer Detection [3] has widely been used in recent years in improving diagnoses and can assist doctors to better approach patient problems.

This paper is organized as follows: related work is discussed in Section II, research methodology is shown in Section III, while the details of the proposed work are illustrated in Section IV. The experimental results are discussed in Section V. Finally, conclusions are shown in Section VI.

\section{RELATED WORK}

Many methodologies and researches have proposed different methods for skin cancer detection [4], especially melanoma skin cancer detection. These methods include Menzies method [5], pattern analysis [6], texture analysis [7]. The complexity related to the poor contrast between the lesion region and skin background, irregular and undulated lesion contour, different colored lesion and several kinds of noise. Image pre-processing has been proposed for image enhancement. Smoothing and de-noising algorithms are usually used in the first process to remove unwanted area such as skin lines, hair, air bubbles and blood vessels.

Segmentation in dermoscopic image automatically presents many difficult tasks. Otsu's segmentation is a thresholding algorithm for automatically converting a greyscale image to a binary image. This algorithm is one of many binarization algorithms, and commonly used for image segmentation. Normalized Otsu's segmentation algorithm proposed by [8], the accuracy given by this algorithm can be used in the further steps to detect the lesion accurately. Texture features are also widely used in melanoma classification where Texture Distinctive Lesion Segmentation (TDLS) extracts the lesion region from the skin [9], [10] which is obtained from dermoscopic image. Delaunay triangulation is used to extract lesion region without the need of training stage, this proposed method obtain high accuracy. [11].

Shape feature is one of major features in image classification. Generally, many researchers use ABCD feature as input to classifier. Percentage of change curvature 
feature [12] is one of shape features which has been presented to classify malignant tumors in mammography images. Finally, for melanoma classification, Naïve Bayes [7], KNN [13], SVM [14] and Deep Neural Network [15] are widely used for improving automatic diagnosis system.

\section{RESEARCH METHODOLOGY}

\section{A. Image Pre-processing}

The important task before segmentation was image pre-processing. Some input images had different contrasts. Hence, image enhancement was used in the first step before feature extraction process. Enhancing contrast using adaptive histogram equalization for adjusting and improving image intensities was different from the original histogram equalization. Adaptive procedure calculated various histograms, which were compatible to explicit regions of the image, and used these values to diffuse the brightness values of the image. Many filters and popular image enhancement methods such as gaussian filter, average filter, median filter, bilateral filter, histogram equalization and gamma correction [12] were used to enhance image quality.

\section{B. Support Vector Machine}

Support Vector Machine (SVM) is specific to supervised learning model which learns or trains from the past input data and makes future predictions as output by teaching the model. SVM is usually used in face detection, text and hypertext categorization, classification of images and bioinformatics. From Fig.1, given 2 labelled classes of data, it acts as a discriminative classifier, formally defined by and optimal hyperplane that separates into two classes. A hyperplane is a decision line or decision surface that splits the space into two parts. Support vectors are the extreme points in the data set and hyperplane which has a maximum distance to the support vectors of any class. The solid line in the middle is the hyperplane and the support vectors have been drawn in dashed lines. To find the largest distance margin, we can get the optimal hyperplane. The optimal hyperplane is the hyperplane that maximizes the distance between the gap or margin of the hyperplane. New data or testing data that are then mapped into the same space can be categorized based on which side of the space they fall.

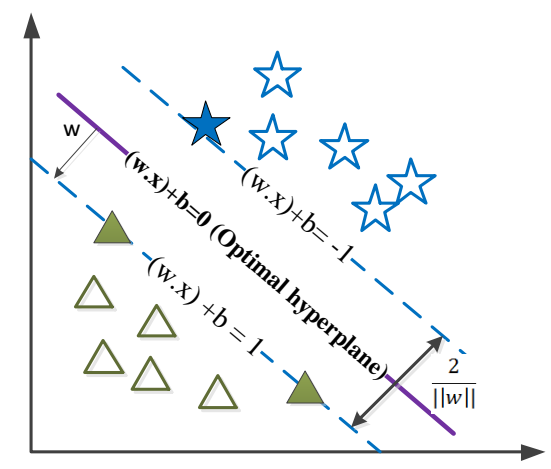

Fig. 1. Support vector machine.

From equation 1, we consider 2 datasets of the form

$$
\left.D=\left\{x_{1}, y_{1}\right), \ldots,\left(x_{l}, y_{l}\right)\right\}, x_{i} \in R^{m}, y_{i} \in\{-1,1\}
$$

where $l$ is the number of dataset, $i$ is the sequence of data, $m$ is number of dimensions, and $y$ is classification result comprising of +1 and -1 to indicate each class of dataset after classification process. In case of linear classification, we consider the dataset by specifying $x_{1}$ to be data of $x_{i}$ and value is +1 . In a similar way, $x_{2}$ is data of $x i$ and value is -1 . This concept can be written as

$$
\begin{aligned}
& \left(w * x_{1}\right)+b>0 \text { where, } y_{i}=+1 \\
& \left(w * x_{2}\right)+b<0 \text { where, } y_{i}=-1
\end{aligned}
$$

where $w$ denotes weight of vector, $x_{1}$ is positive data vector, $x_{2}$ is negative data vector, and $\mathrm{b}$ is bias. Sometimes data is linearly separable, but sometimes it is not linearly separable. The advantage of support vector machine is efficiently performing a non-linear classification using kernels function. Training data can be mapping in a higher dimensional space. There are several kernel functions that can be used in SVM. However, in general purpose, there are some popular kernel functions that we can select to use with SVM.

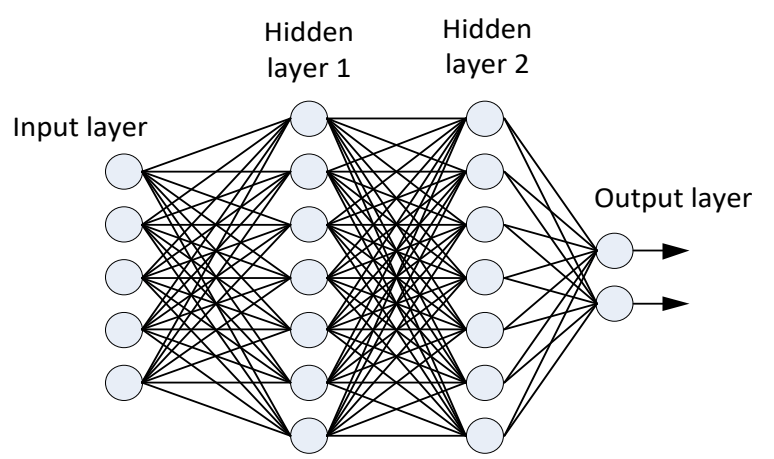

Fig. 2. Deep neural network.

\section{Deep Neural Network}

Deep Neural Network is the method of applying deep neural network which is neural network structures with multiple hidden layers to manipulate problems. Neural network [16] is similar to multilayer perceptron comprised of an input layer, hidden layers and output layer. It's consists of the activation function, weight $(W)$ and biases $(b)$. The output at each node can be demonstrated as

$$
a=f\left(W^{T} x+b\right)
$$

where $f$ denotes the non-linearity function. There are many non-linear functions that are generally used such as hyperbolic, Rectified Linear Unit (RELU) and sigmoid. Deep neural network use multiple hidden layers to learn patterns hierarchically. Activation function in the output layer makes decision to ensure that the output has the probability close to 1. Deep neural network is very popular in medical image processing, due to its performance to classify low level feature such as raw image.

\section{PROPOSED WORK}

In our proposed work, we divided our framework into five major parts: image pre-processing, segmentation, feature extraction, classification and evaluation. 


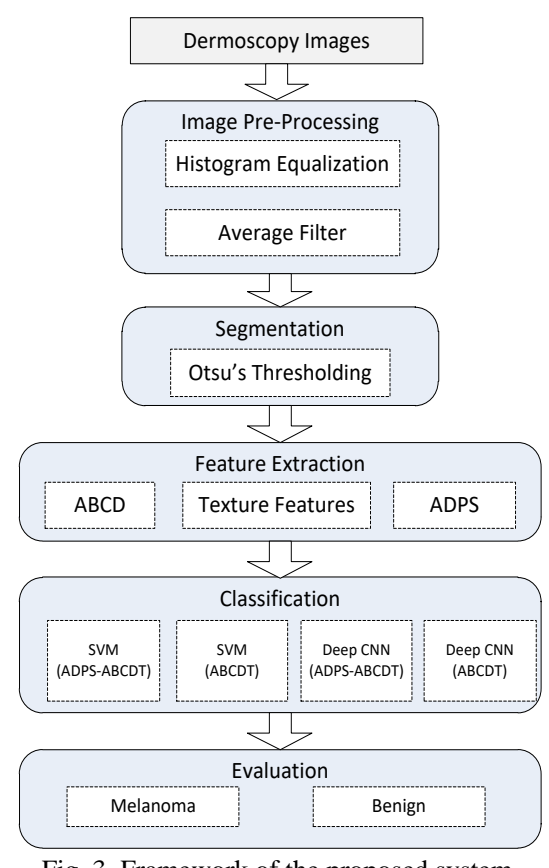

Fig. 3. Framework of the proposed system.

\section{A. Image Segmentation}

Otsu thresholding was used to automatically operate clustering-based image thresholding. This method also converted a grey scale image into binary. The algorithm assumed that the image contained two classes of pixels following bi-modal histogram [17]. Fig. 4 illustrates Otsu thresholding process used to segment lesion from background. After the lesion region had been extracted from the image, many small areas were removed and flood fill method was used to fill little holes in segmented image.

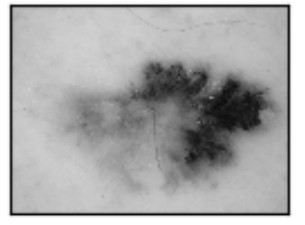

(a)

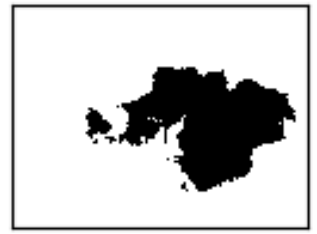

(b)

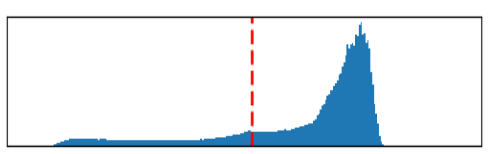

(b)

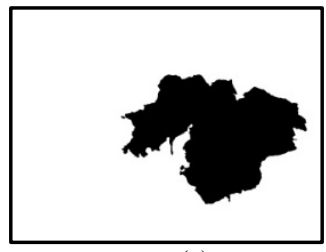

(c)
Fig. 4. Otsu thresholding. (a) original image. (b) histogram. (c) lesion region. (d) remove small areas \& flood fill.

\section{B. Feature Extraction}

Several features were used in feature extraction process. Especially the standard features for diagnosis of melanoma tumor, namely ABCD. Furthermore, texture features and adaptive additional shape features were also used as input in training and testing process.

\section{ABCD Features}

The different properties of shape between melanoma contour and benign contour have been used in feature extraction process. Normally, melanoma contour has notched border or irregular border because of growth of abnormal skin tissues, while benign contour has a rather smooth border. In this process we used the well-known shape features which are normally used in visual inspection, A (asymmetry), B (irregular border) and D (Diameter) as features to input into classifier. In addition, $\mathrm{C}$ or color variegation is also important in classification process. Because several colors such as blue, black, brown, red, white, or the rough distributions of color can probably be a warning sign of melanoma. Benign moles are usually a single shade of brown or tan [18].

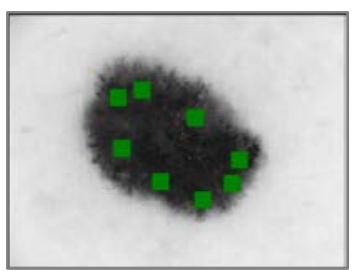

(a)

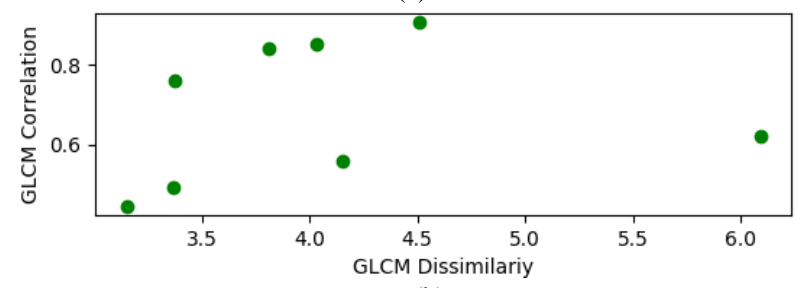

(b)

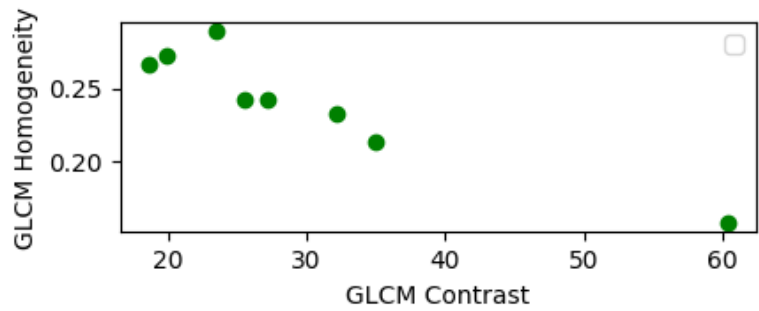

(c)

Fig. 5. GLCM feature. (a) selected pathch. (b) correlation and dissimilarity. (c) homogeneity and contrast.

\section{Texture Features}

Texture features is one of the significant features used in identifying melanoma in lesion image. The Grey Level Co-occurrence Matrix (GLCM) function calculate how often different or similar combination of grey pixel with specific values and in a specified spatial relationship occur in an image. In this work we created GLCM and extracted statistical features which were obtained from the matrix such as correlation, similarity, dissimilarity and contrast, two directions calculation of GLCM were extracted, which were horizontal and vertical direction. Fig. 5 illustrates the statistic features of GLCM which consist of correlation, dissimilarity, homogeneity and contrast.

\section{E. Adaptive Additional Shape Features}

In this proposed work, we extracted shape feature using the percentage change of curvature [12]. First, we automatically found centroid using the properties of binary image such as area and center of mass, from centroid drag lines to every edge pixel along every angles and measure distance from centroid to every edge pixel. After that, plotted the graph with every angle along the $\mathrm{x}$-axis $(0-360$ degrees $)$ and normalized distance on the y-axis. Fig. 7-8, illustrates difference of curvature due to the distinct shape of melanoma and benign tumor. Normalization process was used to get the 
percentage change of curvature. Consequently, the different percentage change of curvature between melanoma and benign was obtained. Many serrates appeared along the edge of melanoma contour and the percentage change of peak became higher. In the opposite, benign tumor had less serrates than malignant contour. Fig. 6 demonstrates the case of changing curvature magnitude. Fig. 7-8 illustrates graphs comparison between malignant and benign.

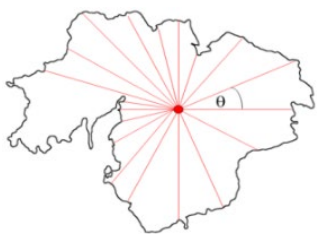

(a)

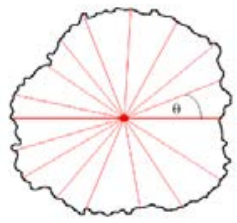

(b)
Fig. 6. Measuring the distance between centroid and edge contour. (a) melanoma. (b) benign.

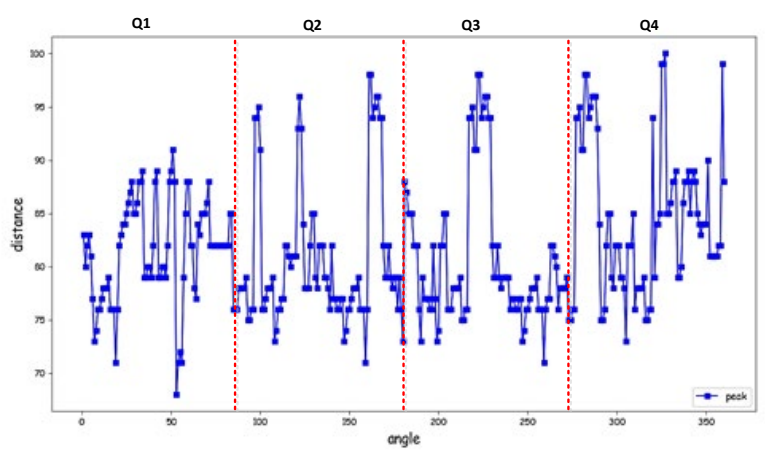

Fig. 7. Graph of curvature: melanoma contour.

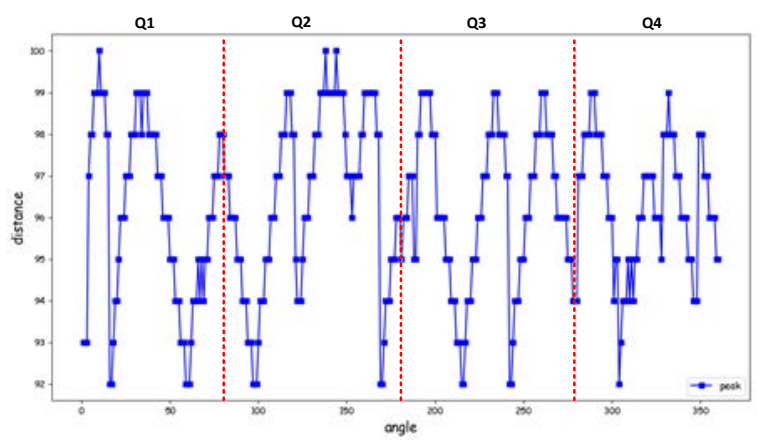

Fig. 8. Graph of curvature: benign contour.

Instead of using every percentage change of curvature along overall angles $(0-360)$ as additional features as the previous work [10], we separated angle into four quadrants: Q1 (0 - 90), Q2 (91 - 180), Q3 (181 - 270) and Q4 (271 $360)$, because we found that too many features were decreasing learning performance and increasing computation complexity, a feature which this research will refer to as Adaptive Additional Shape Features (ADPS). ADPS used only 4 additional features which already compute the percentage change of curvature within each quadrant in average.

\section{F. Classification Using Deep Learning and SVM}

The model first had to be trained; which patterns were associated with melanoma or benign mole. That required labelling the benign versus melanoma patterns, which had to be done by trained features. The features of skin lesion that we extracted from the feature extraction process were input into deep neural network for training dataset. The 24 features that we obtained from feature extraction process comprised of ABCD features (asymmetry (1), notch border (4), colors (5) and diameter (2)), texture features (h-correlation, h-dissimilarity, h-contrast, h-homogeneity, v-correlation, v-dissimilarity, v-contrast, v-homogeneity) and ADPS (Q1, Q2, Q3, Q4).

In this work we used deep neural network and SVM as classifiers. In the training process 500 images including 240 melanoma cases and 260 benign cases were used as input data. For deep neural network 2 hidden layers were used to create classifier. Furthermore, RBF kernel was as a kernel function in SVM.

\section{EXPERIMENTAL RESULTS}

In this research, we have selected 500 images that included both cases of tumor, that are melanoma and benign from The International Skin Imaging Collaboration (https://www.isic-archive.com), which consisted of 240 of melanoma and 260 of benign for input data in training process. In the testing process 120 images were used to evaluate model, which consisted of 55 melanoma images and 65 for benign images.

Table I-IV show the achieved results: the features which produced by Adaptive Additional Shape feature (ADPS) had capability to obtain good result in classification process, both SVM and Deep-NN. In Table I-IV, True Positive (TP) is number of correct classification as melanoma, True Negative (TN) is number of correct classification as benign, False Positive (FP) is number of incorrect classification as melanoma and False Negative is number of incorrect classification as benign.

For evaluation process, accuracy, sensitivity, specificity, precision and f-measure were used. Accuracy is the ratio of correct classifications to the overall amount of classification. Sensitivity or Recall is the ratio of correct positive classification to the overall amount of positive classification. Specificity is the ratio of correct negative classification to the overall amount of negative classification, and F-measure is the ratio of correct classification to the overall amount of positive classification

TABLE I: DEEP NN WITH ADPS-ABCDT

\begin{tabular}{|c|c|c|c|c|}
\hline \multicolumn{2}{|c|}{$\begin{array}{c}\text { Deep NN } \\
\text { (ADPS-ABCDT) }\end{array}$} & \multicolumn{2}{c|}{ Actual } & \\
\cline { 2 - 4 } Predict & Positive & Positive & Negative & \multirow{2}{*}{$\begin{array}{c}\text { Accuracy } \\
=90\end{array}$} \\
\cline { 2 - 4 } & Negative & FN $=6$ & FP $=5$ & \\
\hline \multicolumn{2}{|c|}{ Precision $=90.91 \%$} & $\begin{array}{c}\text { Sensitivity } \\
=89.29 \%\end{array}$ & $\begin{array}{c}\text { Specificity } \\
=92.19 \%\end{array}$ & $\begin{array}{c}\text { F-measure } \\
=90.09 \%\end{array}$ \\
\hline
\end{tabular}

TABLE II: DEEP NN WITH ABCDT

\begin{tabular}{|c|c|c|c|c|}
\hline \multicolumn{2}{|c|}{$\begin{array}{c}\text { Deep NN } \\
\text { (ABCDT) }\end{array}$} & \multicolumn{2}{c|}{ Actual } & \\
\cline { 2 - 4 } Predict & Positive & Positive & Negative & \multirow{2}{*}{$\begin{array}{c}\text { Accuracy } \\
=49\end{array}$} \\
\cline { 2 - 4 } & Negative & FN $=10$ & FP $=6$ & \\
\hline \multicolumn{2}{|c|}{ Precision $=89.09 \%$} & $\begin{array}{c}\text { Sensitivity } \\
=83.05 \%\end{array}$ & $\begin{array}{c}\text { Specificity } \\
=90.16 \%\end{array}$ & $\begin{array}{c}\text { F-measure } \\
=85.96 \%\end{array}$ \\
\hline
\end{tabular}

TABLE III: SVM WITH ADPS-ABCDT

\begin{tabular}{|c|c|c|c|c|}
\hline \multicolumn{2}{|c|}{$\begin{array}{c}\text { SVM } \\
\text { (ADPS-ABCDT) }\end{array}$} & \multicolumn{2}{c|}{ Actual } & \\
\cline { 2 - 4 } Predict & Positive & Positive & Negative & \multirow{2}{*}{$\begin{array}{c}\text { Accuracy } \\
=\end{array}$} \\
\cline { 2 - 4 } & Negative & $\mathrm{FN}=7$ & $\mathrm{FP}=6$ & \\
\hline \multicolumn{2}{|c|}{ Precision $=89.09 \%$} & $\begin{array}{c}\text { Sensitivity } \\
=87.50 \%\end{array}$ & $\begin{array}{c}\text { Specificity } \\
=90.63 \%\end{array}$ & $\begin{array}{c}\text { F-measure } \\
=88.29 \%\end{array}$ \\
\hline
\end{tabular}


TABLE IV: SVM WITH ABCDT

\begin{tabular}{|c|c|c|c|c|}
\hline \multicolumn{2}{|c|}{$\begin{array}{c}\text { SVM } \\
\text { (ABCDT) }\end{array}$} & \multicolumn{2}{c|}{ Actual } & \\
\cline { 2 - 4 } & Positive & Negative & $\begin{array}{c}\text { Accuracy } \\
=84.17 \%\end{array}$ \\
\hline \multirow{2}{*}{ Predict } & Positive & $\mathrm{TP}=46$ & $\mathrm{FP}=9$ & \\
\cline { 2 - 4 } & Negative & $\mathrm{FN}=10$ & $\mathrm{TN}=55$ & \\
\hline \multicolumn{2}{|c|}{ Precision = 83.64\% } & $\begin{array}{c}\text { Sensitivity } \\
=82.14 \%\end{array}$ & $\begin{array}{c}\text { Specificity } \\
=85.94 \%\end{array}$ & $\begin{array}{c}\text { F-measure } \\
=82.88 \%\end{array}$ \\
\hline
\end{tabular}

TABLE V: CLASSIFICATION WITH SVM AND DEEP NN

\begin{tabular}{|c|c|c|c|c|c|}
\hline $\begin{array}{c}\text { Classifi } \\
\text { er }\end{array}$ & Features & $\begin{array}{c}\text { Accurac } \\
\mathbf{y}\end{array}$ & $\begin{array}{c}\text { Sensitivi } \\
\text { ty }\end{array}$ & $\begin{array}{c}\text { Specific } \\
\text { ity }\end{array}$ & $\begin{array}{c}\text { F-mea } \\
\text { sure }\end{array}$ \\
\hline \multirow{2}{*}{$\begin{array}{c}\text { Deep-N } \\
\text { N }\end{array}$} & $\begin{array}{c}\text { ADPS-AB } \\
\text { CDT }\end{array}$ & $\mathbf{9 0 . 8 3 \%}$ & $\mathbf{8 9 . 2 9 \%}$ & $\mathbf{9 2 . 1 9 \%}$ & $\begin{array}{c}\mathbf{9 0 . 0 9} \\
\mathbf{\%}\end{array}$ \\
\cline { 2 - 6 } & ABCDT & $86.67 \%$ & $83.05 \%$ & $90.16 \%$ & $\begin{array}{c}85.96 \\
\%\end{array}$ \\
\hline \multirow{2}{*}{ SVM } & $\begin{array}{c}\text { ADPS-AB } \\
\text { CDT }\end{array}$ & $89.17 \%$ & $87.50 \%$ & $90.63 \%$ & $\begin{array}{c}88.29 \\
\%\end{array}$ \\
\cline { 2 - 6 } & ABCDT & $84.17 \%$ & $82.14 \%$ & $85.94 \%$ & $\begin{array}{c}82.88 \\
\%\end{array}$ \\
\hline
\end{tabular}

\section{CONCLUSIONS}

In this paper, classification of melanoma considering the adaptive additional shape feature is proposed. In feature extraction process we use three types of feature which are $\mathrm{ABCD}$, texture features and adaptive additional shape features. Proposing the combination ADPS and ABCDT in our framework is the major contribution of this research. ADPS is the feature extraction process which can be combined with another feature such as the ABDC and texture feature. The combination of these features outperforms the highest accuracy when using in classification process. The classification accuracy of deep neural network and SVM using all three features, especially when ADPS feature is added is $(90.83 \%$ and $89.17 \%)$ higher than the other $(86.67 \%$ and $84.17 \%)$.

\section{REFERENCES}

[1] Very Wel Health. (2018). How Can You Tell if It's a Mole or Skin Cancer. [Online]. Available: https://www.verywellhealth.com/ moles-vs-melanoma-skin-cancer-identification-gallery-3010833/

[2] R. P. Braun, H. S. Rabinovitz, M. Oliviero, A. W. Kopf, and J. H. Saurat, "Dermoscopy of pigment skin lesions," Dermatol, vol. 52, no. 1, pp. 109-121.

[3] S. Jain, V. Jagtab, and N. Pise,. "Computer aided melanoma skin cancer detection using image processing," in Proc. of International Conference on Intelligent Computing, Communication \& Convergence, 2015, pp. 735-740.

[4] E. Okur and M. Turkan, "A survey on automated melanoma detection," Engineering Applications of Artificial Intelligence, vol. 73, pp. 50-67, 2018.

[5] S. W. Menzies, C. Ingvar, K. A. Crotty, and W. H. McCarthy, "Frequency and morphologic characteristics of invasive melanomas lacking specific surface microscopic features," Archives of Dermatology, vol. 132, no. 10, pp. 1178-1182, 1996.

[6] A. Saez, B. Acha, and C. Serrano, "Pattern analysis in dermoscopic images," Computer Vision Techniques for Diagnosis of Skin Cancer, Berlin: Springer, 2014, pp. 23-48.

[7] I. Maglogiannis and C. N. Doukas, "Overview of advanced computer vision systems for skin lesions characterization,” IEEE Transaction on Information Technology in Biomedicine, vol. 13, no. 5, pp. 721-733, 2009.

[8] S. Y. Kim and S. J. Yun, "Cutaneous melanoma in asians," ChonNam Medical Journal, vol. 52, no. 3, pp. 185-193, 2016.

[9] N. Alfed and F. Khelifi, "Bagged textural and color features for melanoma skin cancer detection in dermoscopic and standard images," Expert Systems with Applications, vol. 90, pp. 101-110, 2017.

[10] A. Santy and R. Joseph, "Melanoma detection using statistical texture distinctiveness segmentation," International Journal of Computer Application, vol. 127, no. 15, pp. 1-5, 2015.
[11] A. Pennisi, D. Bloisi, D. Nardi, A. R. Giampetruzzi, C. Mondio, and A. Facchiano, "Skin lesion image segmentation using delaunay triangulation for melanoma detection," Computerized Medical Imaging and Graphics, vol. 52, pp. 89-103, 2016.

[12] K. Chaiyakhan, N. Kerdprasop, and K. Kerdprasop, "Mammography image classification and clustering using support vector machine and k-means," An International Journal of Research and Surveys, vol. 7, no. 5, pp. 961-967, 2016.

[13] L. Ballerini, R. B. Fisher, B. Aldridge, and J. Rees, "A color and texture based hierarchical K-NN approach to the classification of non-melanoma skin lesion," Color Medical Image Analysis, Berlin: Springer, 2013, pp. 63-86.

[14] N. Situ, T. Wadhawan, X. Yuan, and G. Zouridakis, "Modeling spatial relation in skin lesion images by the graph walk kernel," in Proc. of Annual International Conference of IEEE Engineering in Medical and Biology Society (EMBC), 2010, 6130-6133.

[15] A. Krizhevsky, I. Sutskever, and G. E. Hinton, "Imagenet classification with deep convolutional neural network," Advances in Neural Information Processing Systems, vol. 25, no. 2, pp. 1097-1105, 2012.

[16] N. N. Sultana and N. B. Puhan. (29 November 2018). Recent deep learning method for melanoma detection: A review. [Online]. Avalible: https://www.researchgate.net/profile/Nazneen_Sultana/publication/

[17] J. Premaladha, M. Lakshmi Priya, S. Sujitha, and K. S. Ravichandran, "Normalised Otsu's segmentation algorithm for melanoma diagnosis, Indian Journal of Science and Technology, vol. 8, no. 22, pp. 1-6, 2015.

[18] Melanoma Research Foundation. (5 December 2018). ABCDEs of melanoma: A guide to spotting melanoma. [Online]. Available: https://www.melanoma.org/sites/default/files/u13882/AB CDEs\%20of\%20Melanoma_0.pdf

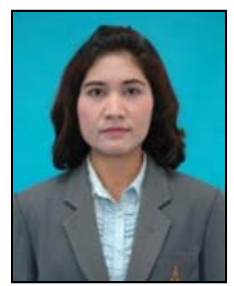

K. Chaiyakhan is currently a faculty member of the Computer Engineering Department, Raja-mangala University of Technology Isan, Thailand. She received her bachelor degree in Computer Engineering from Rajamangala University of Technology Thanya-buri, Thailand, in 1998, master degree in Computer Engineering from King Mongkut's University of Technology Thonburi, Thailand, in 2007, and doctoral degree in Computer Engineering from Suranaree University of Technology, Thailand, in 2016. Her current research includes data mining, machine learning, image classification and image clustering

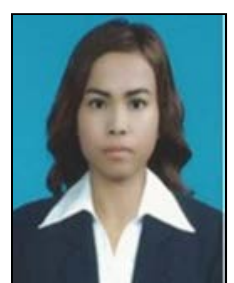

P. Chujai is a lecturer at the Electrical Technology Education Department, Faculty of Industrial Education and Technology, King Mongkut's University of Technology Thonburi, Thailand. She received her bachelor degree in Computer Science from Ramkhamhaeng University, Thailand, in 2000 master degree in Computer and Information Technology from King Mongkut's University of Technology Thonburi, Thailand, in 2004 and doctoral degree in Computer Engineering, Suranaree University of Technology, Thailand, in 2015. Her current research includes Ontology, Recommendation System, Time Series, and Imbalanced data classification

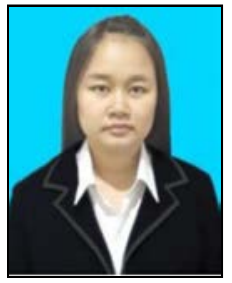

R. Chanklan is a teaching assistant at the School of computer engineering, Suranaree University of Technology, Thailand. She received his bachelor degree in Computer Engineering from Suranaree University of Technology, Thailand, in 2013, the master degree in computer engineering from Suranaree University of Technology, Thailand, in 2014. Her current research of interest includes classification, data mining, artificial intelligence.

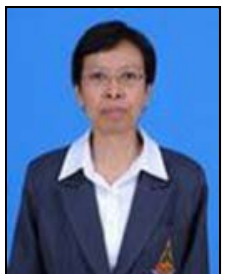

N. Kerdprasop is an associate professor at the School of Computer Engineering, Suranaree University of Technology, Thailand. She received her bachelor degree in Radiation Techniques from Mahidol University, Thailand, in 1985, master degree in Computer Science from the Prince of Songkla University, Thailand, in 1991, and doctoral degree in Computer Science from Nova Southeastern University, U.S.A, in 1999. Her research of interes includes knowledge discovery in databases, artificial intelligence, logic and intelligent databases. 